\title{
Chronic Urate Nephropathy
}

National Cancer Institute

\section{Source}

National Cancer Institute. Chronic Urate Nephropathy. NCI Thesaurus. Code C123013.

Chronic kidney disease resulting from deposition of urate crystals or microtophi in the medullary interstitium. 